\title{
ELECTRON PARAMAGNETIC RESONANCE SPECTROMETRY OF PHOTOSYSTEM I MUTANTS IN BARLEY
}

\author{
by \\ BIRGER LINDBERG MØLLER \\ Department of Physiology, Carlsberg Laboratory \\ Gamle Carlsberg Vej 10, DK-2500 Copenhagen Valby \\ and \\ JONATHAN H. A. NUGENT and MICHAEL C. W. EVANS \\ Department of Botany and Microbiology, \\ University College London, Gower Street, London WC1E 6BT
}

Keywords: Photosystem I particles, thylakoid polypeptides

Two photosystem I mutants from barley have been characterized by EPR spectroscopy. The mutant viridis$z b^{63}$ is devoid of photosystem I activity and lacks the EPR signals corresponding to the photosystem I reaction center $P 700$ and the acceptors $\mathrm{A}_{1}, \mathrm{X}(=\mathrm{A} 2), \mathrm{B}$, and $\mathrm{A}$. The mutant viridis- $n^{34}$ shows an eighty percent decrease in photosystem I activity and contains low levels of the above centres. The EPR signals P700, $A_{I}, X, B$, and $A$ were present in a photosystem I particle containing only three polypeptides with molecular weights of 110,000 , 18,300 , and 15,200. The same three polypeptides plus an additional polypeptide with a molecular weight of 14,800 were absent or decreased in viridis-z $b^{63}$ and viridis $-n^{34}$, respectively. A photosystem II signal normally masked by $P 700$ was detected in the mutants.

\section{INTRODUCTION}

Mutants deficient in a single or a few of the thylakoid polypeptides involved in photosynthetic electron transport are important tools for the identification of new components in the photosynthetic apparatus $(11,12,15)$ and for the allocation of spectroscopically defined components to specific polypeptides $(9,17,27)$. One of the spectroscopic techniques which have been most successful in the detection of new components in photosynthetic electron transfer is electron paramagnetic resonance spectrometry

Abbreviations: $\mathrm{BSA}=$ bovine serum albumin; DTT = dithiothreitol; EPR = electron paramagnetic resonance; SDS $=$ sodium dodecyl sulfate. 
(EPR). This technique enables the detection of paramagnetic molecules. EPR spectra can therefore be obtained from the chlorophyll and quinone radicals associated with the photosynthetic reaction centres and from the components of the electron transport chain containing a transition metal (3). When the EPR spectra are recorded at cryogenic temperatures the light induced photoreactions in the sample are restricted to those in the reaction centres because the electron flow in the intermediary chain is blocked.

EPR spectrometry of photosystem I in plants has revealed the presence of two bound ironsulfur centres A and B (3). When centres A and $B$ have been chemically reduced by dithionite, illumination of photosystem I particles (which properly should be called vesicles) at cryogenic temperatures results in a reversible oxidation of $P 700$ and reduction of a component $\mathrm{X}$ which probably also is an iron-sulfur centre $(8,26)$. Optical studies (14) and EPR studies involving illumination of photosystem I particles in which centres $\mathrm{A}, \mathrm{B}$, and $\mathrm{X}$ were already reduced by dithionite (7) revealed the existence of an additional acceptor $\mathrm{A}_{1}$ located between $P 700$ and $X$. The exact nature of this component is not known but it may be a pheophytin molecule or a special chlorophyll radical (8). In this study we report the EPR characteristics of mutants in barley which are deficient in or lack photosystem I.

\section{MATERIALS AND METHODS}

\subsection{Plant material}

Seeds of wild-type barley (Hordeum vulgare cv. Svalof's Bonus) and the two recessive mutants viridis $-n^{34}$ and viridis $-z b^{63}$ were germinated in vermiculite moistened with tap water. Seedlings were harvested after growing for 7 days at $20^{\circ} \mathrm{C}$ in continuous white light $(1700$ lux). The mutant seedlings were separated from the wild-type phenotypes by their slightly paler green color. Both mutants are seedlings lethals and are therefore maintained in the heterozygous state.

\subsection{Chloroplast and thylakoid isolation}

Chloroplasts were prepared by homogenizing precooled leaves in a medium composed of 0.33 M-sorbitol, $20 \mathrm{~mm}$-sodium pyrophosphate, 5 $\mathrm{mm}-\mathrm{MgCl}_{2}$ and $2 \mathrm{~mm}$-sodium ascorbate at $\mathrm{pH}$ 6.5. The homogenate was filtered through two layers of $31 \mu \mathrm{m}$ nylon gauze and centrifuged at $4,000 \mathrm{~g}$ for $5 \mathrm{~min}$. The chloroplast pellet was resuspended and washed in $50 \mathrm{~mm}$-tricine, 0.2 $\mathrm{m}-\mathrm{KCl}, 0.5 \mathrm{~mm}-\mathrm{DTT}$ and $0.05 \% \mathrm{BSA}$ at $\mathrm{pH} 7.6$. The thylakoid pellet obtained after centrifugation for $5 \mathrm{~min}$ at 4,000 $\mathrm{g}$ (wild-type) or $8,000 \mathrm{~g} \mathrm{(mu-}$ tants) was used for the EPR measurements.

\subsection{Photosystem I particles}

Photosystem I particles were prepared as described in (16).

\subsection{Chlorophyll determination}

Chlorophyll was determined as described in (1).

\subsection{Electron paramagnetic resonance spectra}

EPR measurements were made using a Jeol JES Fel-X X band spectrometer. Samples were maintained at cryogenic temperatures using an Oxford Instruments liquid helium cryostat. A $1000 \mathrm{~W}$ projector was used for illumination of samples. Spectra were recorded into a Tektronix 4051 computer which was also used to plot the spectra shown. g-Values were calculated using an external standard of $\mathrm{Mn}^{2+}$ and were checked internally using the g-value of 2,0048 for signal II. g-Values and field scales indicated on the figures are approximate.

\section{RESULTS}

\subsection{Polypeptide composition of barley thylakoids and photosystem I particles}

The polypeptide compositions of the various preparations used in this study have been published earlier $(9,16)$ and are summarized in Figure 1 for convenience.

\subsection{Photosystem I acceptors}

The EPR spectrum of wild-type thylakoids reduced by $10 \mathrm{~mm}$ ascorbate and frozen in the dark (Figure 2A) demonstrates a line at $\mathrm{g}=$ 


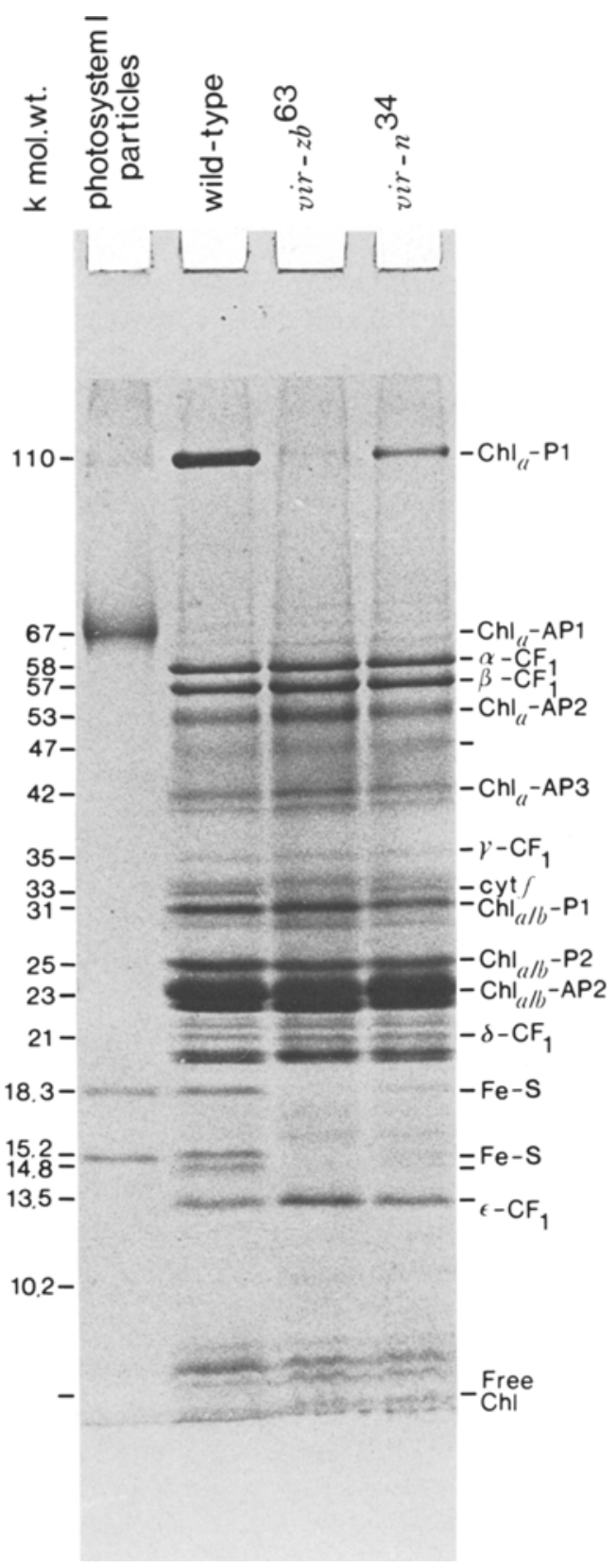

Figure 1. Thylakoid polypeptide pattern of wild-type and mutant barley compared with that of photosystem I particles.

The same amount of chlorophyll was applied to each slot and the separation was carried out at $19^{\circ} \mathrm{C}$ on an $11-15 \%$ SDS-polyacrylamide gradient gel. The molecular weights assigned to the polypeptides in the low molecular weight region are inaccurate and only given as guide.

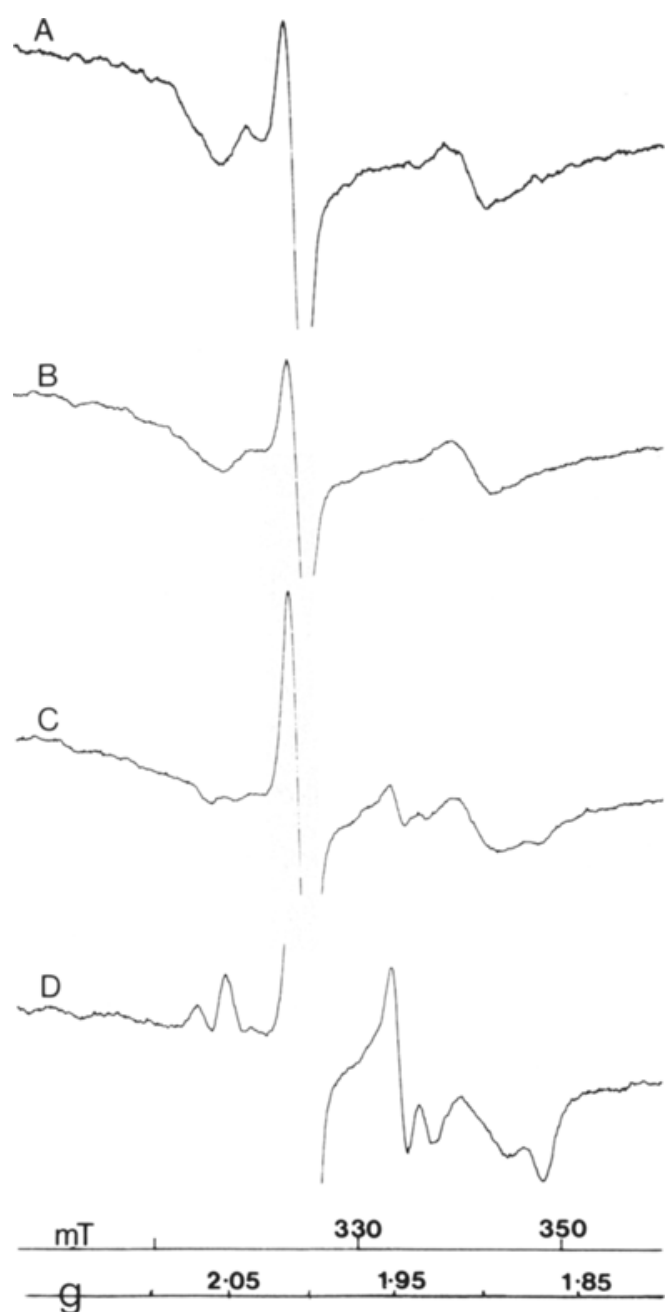

Figure 2. EPR spectra showing iron-sulfur centres in barley chloroplasts.

A, wild-type chloroplasts frozen after dark adaptation in the presence of $10 \mathrm{~mm}$-ascorbate. $\mathrm{B}$, viridis$n^{34}$ chloroplasts treated as in A. C, sample in B after illumination at $14 \mathrm{~K}$. D, sample in A after illumination at $14 \mathrm{~K}$. Chlorophyll concentration $4 \mathrm{mg} \cdot \mathrm{ml}^{-1}$, microwave power $10 \mathrm{~mW}$, gain 500 , temperature $15 \mathrm{~K}$, modulation amplitude $1 \mathrm{mT}$, frequency 9.11 $\mathrm{GHz}$, scan rate $25 \mathrm{mT} \cdot \mathrm{min}^{-1}$, time constant $0.1 \mathrm{sec}$.

1.89 due to the reduced Rieske iron-sulfur centre functioning as an electron carrier component between photosystem II and I (12). The line at $\mathrm{g}$ 2.03 may also be due to the Rieske centre (25) but in our experiments exhibits different EPR characteristics suggesting an unidentified compo- 
nent. The latter signal is superimposed on a line due to $\mathrm{Cu}^{2+}$. The radical near $\mathrm{g} 2.00$ is termed signal II and originates from a component in photosystem II. A similar EPR spectrum was obtained from chloroplasts of the barley mutant viridis $-n^{34}$ upon the above treatment (Figure 2B). This demonstrates that the mutant has a normal content of the Rieske centre. Illumination of the samples at $15 \mathrm{~K}$ induces a signal due to the oxidation of $P 700$ and signals corresponding to
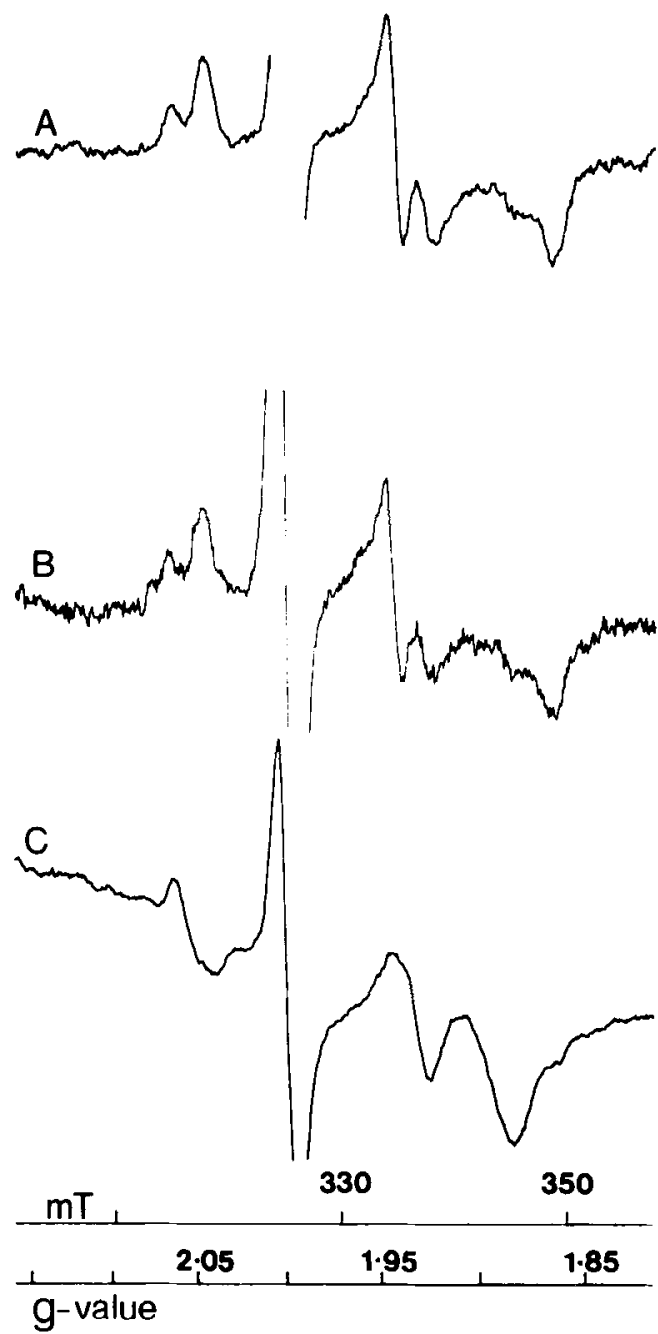

Figure 3. EPR spectrum (A) of wild-type chloroplasts showing light induced signal obtained by subtraction of Figure 1(A) from Figure 1(D) and EPR spectrum (B) of viridis $-n^{34}$ showing light induced signal obtained by subtraction of Figure l (B) from Figure 1 (C) expanded $\times$ 4. C, EPR spectrum of sample in Figure 1 (D) after storage at $77 \mathrm{~K}$ in the dark. EPR conditions as in Figure 2 except gain $10^{3}$. the reduced iron sulfur centre acceptors $A$ and $B$. (Figures 2C and 2D). However, comparison of the spectrum of the mutant (Figure $2 \mathrm{C}$ ) with that of the wild-type indicates the much lower photosystem I content of the mutant. This is in agreement with earlier photochemical measurements (9). The light induced signals in wild-type and viridis $-n^{34}$ chloroplasts can be demonstrated by subtracting the spectrum obtained before illumination from that obtained after illumination (Figures $3 \mathrm{~A}$ and 3B). The light induced difference spectrum of viridis $-n^{34}$ has been expanded 4 times to give approximately equal spectral sizes of centre A and measurements on the spectra indicate that the low temperature photochemical activity of photosystem I in viridis $-n^{34}$ is approximately $20 \%$ of that in wildtype. Besides the appearance of the $P 700$ radical, the lineshape observed is attributed to the reduction of iron-sulfur centres A and B. It is apparent that only one of these centres is reduced in each reaction centre as no interaction between centres A and B is observed (23). When the illuminated samples are stored in the dark at $77 \mathrm{~K}$ a preferential decay of the signal corresponding to centre $\mathrm{A}$ is observed (Figure $3 \mathrm{C}$ ). This allows the spectrum of centre $B$ to be seen superimposed on that of the Rieske centre. Whereas at cryogenic temperatures in barley either centre A or centre B can be first reduced (23), in spinach the reduction of centre $B$ is normally seen only in samples where centre $A$ is already reduced $(4,5)$.

The reduction of iron-sulfur centres A and B in the same reaction centre is observed when thylakoid membranes of barley are subjected to strong reducing conditions using dithionite at $\mathrm{pH}$ 10 and illumination during freezing of the sample (Figure 4B). This also results in the reduction of the Rieske centre. The EPR spectrum of photosystem I particles from barley treated under the same conditions likewise shows the reduction of both iron-sulfur centres $A$ and $B$ in the same reaction centre (Figure 4A). Thylakoids of the photosystem I mutant viridis$n^{34}$ were also examined under these highly reducing conditions (Figure $4 \mathrm{C}$ ). The spectrum is expanded two times compared to those in Figure $4 \mathrm{~A}$ and $4 \mathrm{~B}$ and indicates a content of centre $\mathrm{A}$ and $B$ which is only $20-25 \%$ of that of the wildtype level. 

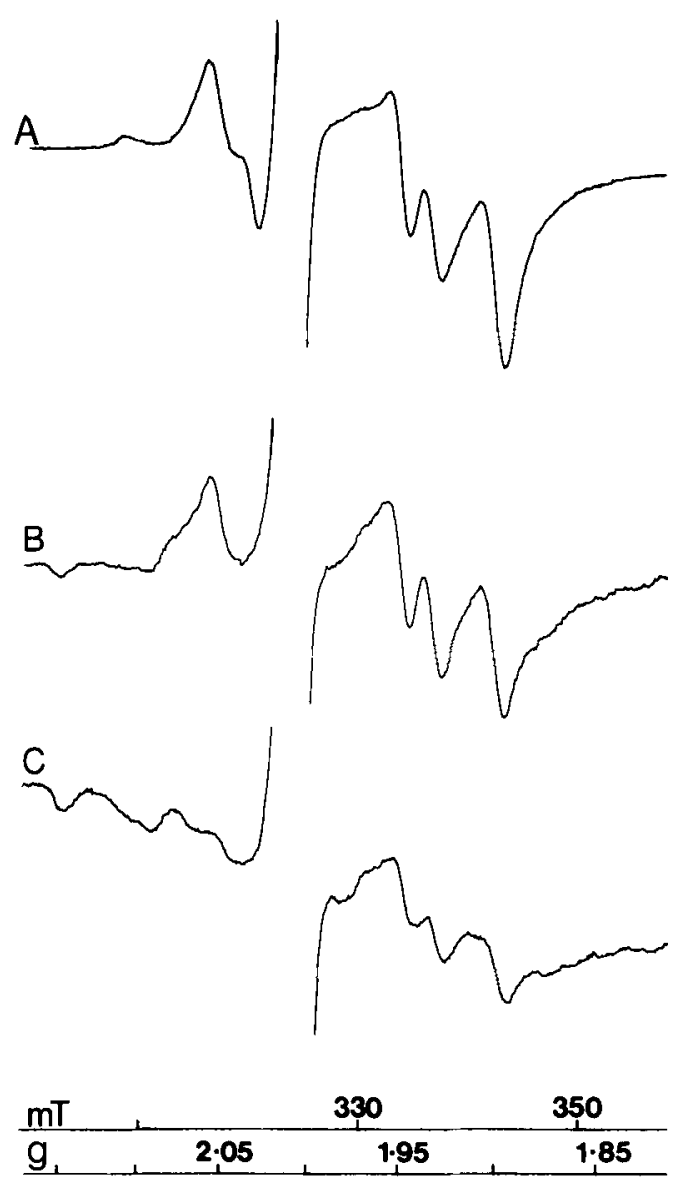

Figure 4. EPR spectra of samples reduced by sodium dithionite for 30 minutes at $\mathrm{pH} 10$ and then frozen under illumination.

A, photosystem I particles $(3.0 \mathrm{mg}$ chlorophyll $\cdot \mathrm{ml}^{-1}$ ). B, wild-type chloroplasts. C, viridis $-n^{34}$ chloroplasts. Conditions as in Figure 2 except $\mathrm{C}$, gain $10^{3}$.

Optimal conditions for the detection of the two acceptors $A_{1}$ and $X$ are obtained by lowering the temperature of the samples to $8 \mathrm{~K}(6)$. The spectrum of centre $X$ has g-values at 1.78, 1.88 and 2.08 (8). In wild-type chloroplasts, the spectrum of centre $X$ is seen superimposed on that of the reduced centres A and B (Figure 5A). The amount of centre $\mathrm{X}$ in viridis- $n^{34}$ is very small (Figure 5B), again indicating a lower photosystem I content in this mutant. The large radical expressed in both spectra at $\mathrm{g} 2.00$ is probably due to the intermediate acceptor $A_{1}(8)$.

The mutant viridis- $z b^{63}$ shows a complete absence of photosystem I activity as indicated both by fluorescence induction kinetics and in vitro electron transport measurements (9). In agreement with this, thylakoids from this mutant showed no photochemical or chemical reduction of the iron-sulfur centres $\mathrm{A}, \mathrm{B}, \mathrm{X}$ or Rieske and no oxidation of $P 700$. However, signals from iron-sulfur centres of different behavior were observed in this mutant upon chemical reduction (Figure 6). Chemical reduction of one centre is observed at $-250 \mathrm{mV}$ (Figure 6B) and additional reduced centres are seen after poising at $-430 \mathrm{mV}$ (Figure $6 \mathrm{C}$ ). The peak g-values are 1.942, 1.920,1.886 and 1.863. The line-shape, redox behavior and g-values are characteristic of the iron-sulfur centres from mitochondrial NADH dehydrogenase (24). Further support for this assignment was obtained by the observation of one of these centres at $77 \mathrm{~K}$ (Figure 6E) which is a property of the $2 \mathrm{Fe}-2 \mathrm{~S}$ centres in NADH dehydrogenase (24). To investigate whether these signals were of mitochondrial origins, mutant thylakoids were puri-

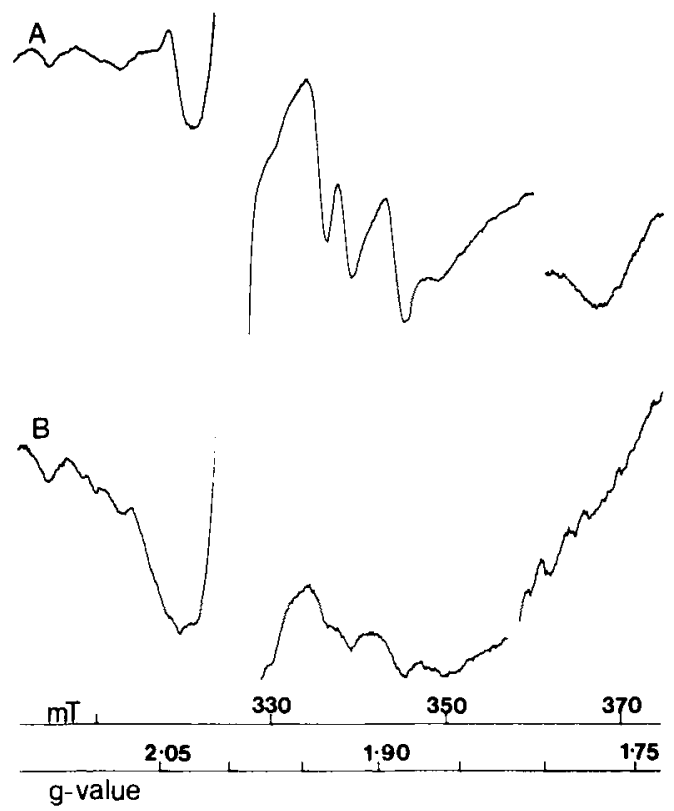

Figure 5. EPR spectra of samples reduced by sodium dithionite for 30 minutes at $\mathrm{pH} 10$ and then frozen under illumination.

A, wild-type chloroplasts. B, viridis- $n^{34}$ chloroplasts. Conditions as in Figure 2 except microwave power $20 \mathrm{~mW}$, temperature $8 \mathrm{~K}$, instrument gain in $\mathrm{A}, 500$, and in $\mathrm{B} 2 \times 10^{3}$. In the region of $\mathrm{g} 1.8$ the gains are $10^{3}$, and $4 \times 10^{3}$, respectively. 


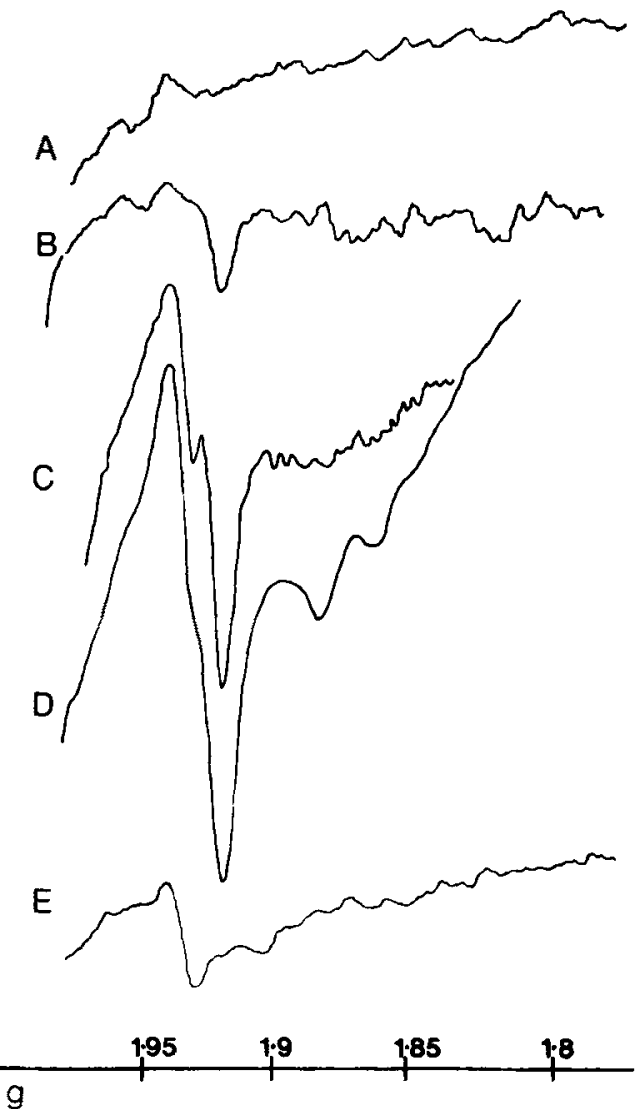

Figure 6. Chloroplasts of viridis- $2 b^{63}$ poised (A) at 0 $\mathrm{mV}$ and (B) at $-250 \mathrm{mV}$.

Chlorophyll concentration $1 \mathrm{mg} \cdot \mathrm{ml}^{-1}$, microwave power $20 \mathrm{~mW}$, gain $2.5 \times 10^{3}$, temperature $15 \mathrm{~K}$, modulation amplitude $1 \mathrm{mT}$, frequency $9.11 \mathrm{GHz}$, scan rate $50 \mathrm{mT} \cdot \mathrm{min}^{-1}$, time constant $0.1 \mathrm{sec}$. (C) at $-430 \mathrm{mV}$. Chlorophyll concentration $3 \mathrm{mg} \cdot \mathrm{ml}^{-1}$, microwave power $10 \mathrm{~mW}$, gain $5 \cdot 10^{3}$, scan rate 25 $\mathrm{mT} \cdot \mathrm{min}^{-1}$, time constant $0.3 \mathrm{sec}$, modulation amplitude $0.63 \mathrm{mT}$. (D) as (C) but gain $2.5 \times 10^{3}$, time constant $1 \mathrm{sec}$ and modulation amplitude $1 \mathrm{mT}$. (E) as (C) but temperature $77 \mathrm{~K}$, gain $2 \times 10^{3}$.

fied by flotation on a discontinuous sucrose gradient. This purification procedure produces two green bands. The upper one contains purified thylakoids whereas the lower one contains thylakoids contaminated to a higher extent with mitochondria (10). EPR spectroscopy of these two samples using equal amounts of chlorophyll showed a marked decrease of the signal in the upper band but an approx. ten times enrichment in the lower band. These signals are therefore concluded to have their origin from mitochondrial NADH dehydrogenase.
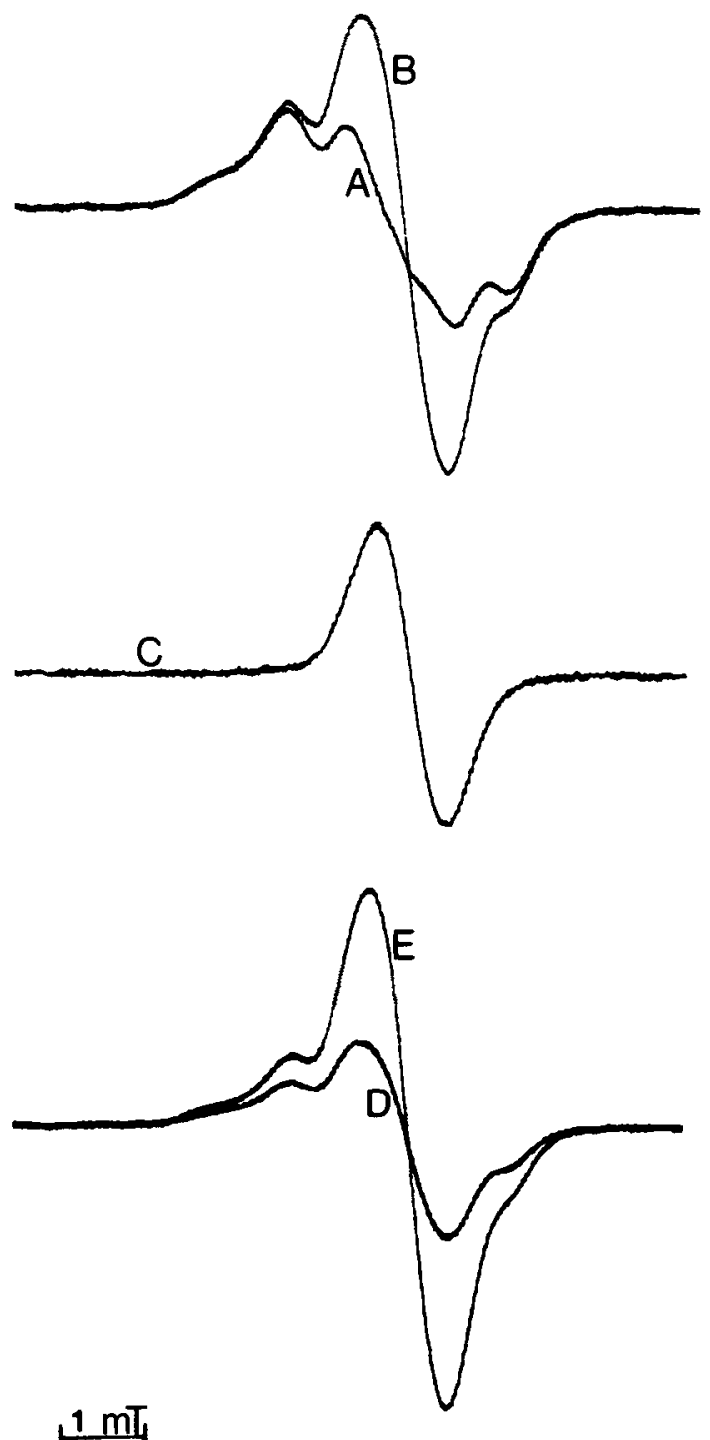

$1 \mathrm{mg}$

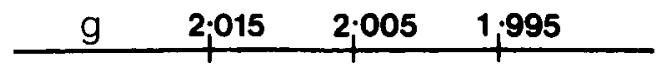

Figure 7. EPR spectra of wild type chloroplasts frozen after dark adaptation for $15 \mathrm{~min}$ (A), after illumination at $77 \mathrm{~K}(\mathrm{~B})$ and difference spectrum of the two (C). The same sample after dark storage at $77 \mathrm{~K}$ for several days is shown in (D) and after a new illumination at $77 \mathrm{~K}$ in (E).

Chlorophyll concentration $2 \mathrm{mg} \cdot \mathrm{ml}^{-1}$, microwave power $100 \mu \mathrm{W}$, temperature $77 \mathrm{~K}$, gain $10^{3}$, modulation amplitude $0.2 \mathrm{mT}$, frequency $8.9 \mathrm{GHz}$, scan rate $5 \mathrm{mT} \cdot \mathrm{min}^{-1}$, time constant $0.3 \mathrm{sec}$. 


\section{3. $P 700$ and other $g$ 2.00 radicals}

The EPR spectrum obtained upon illumination of wild-type thylakoids reveals a large radical signal in the $\mathrm{g} 2.00$ region (Figures 2 and 3). This region was studied in detail by the use of a narrow magnetic field and the spectra were recorded at $77 \mathrm{~K}$ and $100 \mu \mathrm{W}$ to minimize saturation effects on the $\mathrm{g} 2.00$ radicals (Figure 7). No significant differences in behaviour were however observed at lower temperatures. Dark adapted untreated wild-type thylakoids (Figure 7A) show the presence of signal II with a $g$ value of 2.0046 (19). Illumination at $77 \mathrm{~K}$ results in the oxidation of $P 700$ as evident from the increase in signal size (Figure 7B) and from the difference spectrum (Figure 7C). However, the EPR spectrum of $P 700^{+}$has a $g$ value of 2.0026 and a
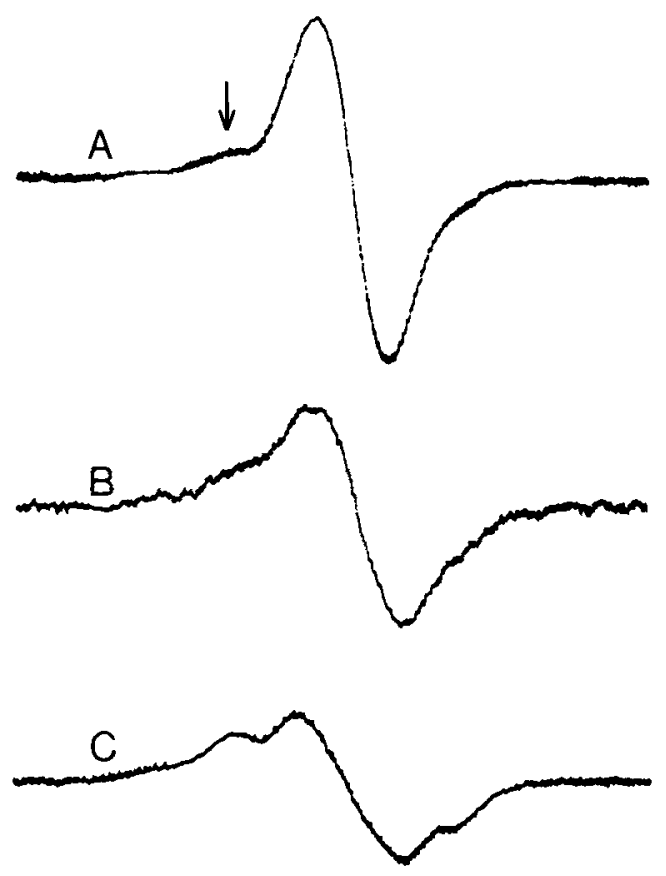

${ }_{1 \mathrm{mT}}$

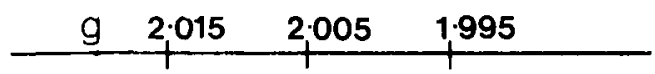

Figure 8. Difference spectra showing light induced signals upon re-illumination of chloroplasts from wild-type $(=$ Figure $7 \mathrm{E}-7 \mathrm{D})$ in $\mathrm{A}$, from viridis $-n^{34}$; in $\mathrm{B}$ and from viridis $-z b^{63}$ in $\mathrm{C}$.

Conditions: (B) chloroplast concentration 4 $\mathrm{mg} \cdot \mathrm{ml}^{-1}(\mathrm{C})$ chloroplast concentration $3 \mathrm{mg} \cdot \mathrm{ml}^{-1}$. Gain $1.25 \times 10^{3}$.

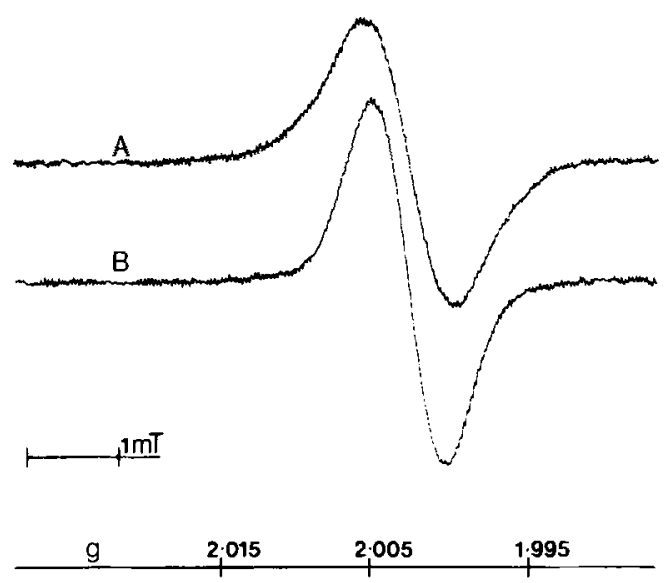

Figure 9. EPR spectra showing the broader linewidth of the light induced radical in viridis-z $z b^{63}$ chloroplasts (A) than in wild-type chloroplasts (B). Samples were reduced by $10 \mathrm{~mm}$-ascorbate and frozen in the dark.

Conditions as in Figure 7 except modulation amplitude $0.1 \mathrm{mT}$, gain 10 in $(\mathrm{A})$ and $2 \times 10^{3} \mathrm{in}(\mathrm{B})$.

peak width of 7.5 gauss whereas the peak width of the signal in the difference spectrum is 8.5 gauss. This widening of the peak is brought about by a superimposed photosystem II signal as evidenced from the studies with the mutant viridis- $z b^{63}$ described below. When the illuminated sample was stored at $77 \mathrm{~K}$ in the dark, a nonuniform decay of the signals is observed (Figure 7D). Subsequent illumination of the sample at 15K (Figure 7E) restores the signal to a similar shape but with slightly larger size compared to that seen in Figure 7B. In addition to a narrow radical, this second illumination produces a small increase in signal II as shown by the low field peak (arrowed) in the light minus dark difference spectrum (Figure $8 \mathrm{~A}$ ). The increase in signal II upon the second illumination at cryogenic temperature was more easily demonstrated using the mutants viridis- $n^{34}$ (Figure 8B) and viridis- $z b^{63}$ (Figure $8 \mathrm{C}$ ). Samples of the latter mutant showed no EPR signals corresponding to centres $\mathrm{A}$ and $\mathrm{B}$ and no $P 700^{+}$was observed in samples of viridis $-z b^{63}$ frozen under illumination or oxidized with $5 \mathrm{~mm}$-ferricyanide $(21,22)$. The narrow $10 \mathrm{~g}$ component in Figure $8 \mathrm{C}$ must thus originate from photosystem II. The greater linewidth of this component as compared to $P 700^{+}$can be demonstrated by 
comparing the signals produced by low temperature illumination of chloroplasts from viridis$z b^{63}$ (Figure 9A) and from wild-type (Figure 9B). Both samples were frozen in the dark after reduction with $10 \mathrm{~mm}$-ascorbate. Direct comparison of radical sizes in these ascorbate treated samples where the component producing signal II was reduced and signal II therefore absent, gave a simplified situation allowing an approximate calculation of the photosystem II content in the radical signal. This showed that in wild-type chloroplasts, approximately $15 \%$ of the radical is due to the 10 gauss photosystem II radical. This radical has a $g$ value of 2.003 similar to that of $P 700$ and its presence in wild-type chloroplasts is only observed with difficulty as a slight increase in the linewidth of apparent $P 700^{+}$. This radical has therefore escaped detection until the mutants lacking photosystem I were examined. The mutant viridis $-n^{34}$ has about $25 \%$ of the wild-type photosystem I level and the photosystem II radical thus contributes $40 \%$ of the total radical. Accurate comparisons are not possible due to the number of overlapping radical signals and the different content of chlorophyll-proteins in wild-type and mutants.

\subsection{Other paramagnetic centres}

The signal induced by low temperature illumination near g 3.00 is attributed to cytochrome $b-559(13,20)$ and is present in all three chloroplast preparations examined. This confirms the earlier results which suggested that both mutants had normal photosystem II electron transport $(9,17)$. The g 6.00 signal attributed to cytochrome $b-563$ (20) was also present in all three preparations although it appeared to be smaller in viridis-z $b^{63}$. This would again be in agreement with earlier observations (9). However, all the cytochrome signals observed were small and therefore difficult to quantify.

\section{DISCUSSION}

Earlier studies on chloroplast lamellar systems and photosystem I particles permitted assignment of EPR signals to the P700 containing reaction centre of photosystem $I$ and to four photosystem $I$ acceptors denoted $A_{1}, X\left(=A_{2}\right)$,
$\mathrm{B}$, and $\mathrm{A}(3,7,8,14,26)$. The EPR studies here carried out on the mutant viridis $-n^{34}$ revealed a marked decrease in the above signals whereas the signals were absent from viridis- $z b^{63}$. This observation is in agreement with an earlier study (9) which showed that viridis $-n^{34}$ retains only $20 \%$ of the wild-type level of photosystem I whereas viridis $-z b^{63}$ was devoid of any photosystem I activity. Both of these mutants showed normal photosystem II electron transport.

Polypeptide analysis of the two photosystem I mutants showed that four polypeptides with molecular weights of $110,000,18,300,15,200$, and 14,800 were absent from viridis- $z b^{63}$ and markedly decreased in viridis $-n^{34}$ suggesting that these polypeptides are linked to photosystem I (9). If this assignment is correct, these polypeptides should also be present in photosystem I particles showing the EPR signals attributed to photosystem I. Most photosystem I particles earlier prepared have either not been analyzed with respect to polypeptide composition or have shown a multiplicity of polypeptides. These preparations have therefore not been useful for the assignment of the observed EPR signals to individual polypeptides. However, BENGIS and NeLSON (2) prepared a photosystem I particle composed of only six polypeptides with molecular weights of $70,000,25,000,20,000,18,000$, 16,000 and 8,000 . This particle catalyzes plastocyanin dependent photooxidation of cytochrome $f$ and photoreduces $\mathrm{NADP}^{+}$in the presence of ferredoxin and ferredoxin-NADP reductase (2) and produces EPR signals corresponding to $P 700$ and the bound iron-sulfur centres A and B (18). The low molecular weight components of the photosystem I particle were depleted when the preparation was subjected to SDS treatment prior to the sucrose gradient centrifugation step. A corresponding decrease in the EPR signals of the iron-sulfur centres suggested that these polypeptides carried the iron-sulfur centres. However, the detergent treatments necessary to dissociate these polypeptides from the $P 700$ chlorophyll $a$-protein 1 reaction center polypeptide may also have caused the destruction of the iron-sulfur clusters. In this study these ambiguities were overcone by the use of a photosystem I particle prepared by the combined use of digitonin and the zwitterionic detergent Empigen BB (16). This particle contains only three 
polypeptides with molecular weights of 110,000 , 18,300 , and 15,200 and catalyzes light-dependent electron transfer from ascorbate/DCPIP to methylviologen. The EPR studies reveal that this simple photosystem I particle carries the reaction center chlorophyll $P 700$ and the four acceptors $A_{1}, X\left(=A_{2}\right), B$, and $A$. Electrophoretic analyses show that the three constituent polypeptides of the photosystem I particle comigrate with three of the four polypeptides of wild-type thylakoids found to be absent or deficient in the photosystem I mutants. Preliminary labelling experiments indicated the incorporation of ${ }^{59} \mathrm{Fe}$ into the two low molecular weight polypeptides of the photosystem I particle suggesting that these could represent iron-sulfur centres $A$ and B. Most likely, the two acceptors $A_{1}$ and $X$ are located on the 110,000 molecular weight polypeptide chlorophyll $a$-protein 1 also carrying the reaction centre chlorophyll $P 700$.

From earlier data on photosystem I mutants, it was inferred that the photosystem I reaction center $(P 700)$ forms an integral complex with several polypeptides of low molecular weight (9). This is supported by the EPR studies here reported since it was found that the absence of measurable photosystem I activity was not due to the lack of one individual electron acceptor: All acceptors were absent in viridis- $z b^{63}$ and the major signals observed in viridis $-n^{34}$ reached $20 \%$ of the wild-type level. The centre $\mathrm{X}$ is a difficult EPR signal to study and appeared to be decreased in amount rather more than centers $A$ and $B$.

The study of photosystem II by EPR spectroscopy has been hampered by the presence of relatively large superimposed signals derived from photosystem I components and by the difficulties encountered in preparing photosystem II particles devoid of photosystem I signals. The mutant viridis $-z b^{63}$ is therefore an excellent starting material for such investigations and permitted the detection of a 10-12 gauss wide photosystem II radical previously masked by $P 700^{+}$.

\section{ACKNOWLEDGEMENTS}

Professor D. voN WetTsteIN is thanked for encouragement throughout this work. The work was supported by the Commission of the
European Communities, contracts No. ESD013-DK and ESD-014-UK of the Solar Energy Program.

\section{REFERENCES}

1. ARnon, D. I.: Copper enzymes in isolated chloroplasts. Polyphenoloxidase in Beta vulgaris. Plant Phys. 24, 1-14 (1949)

2. Bengis, C. \& N. Nelson: Purification and properties of the photosystem I reaction center from chloroplasts. J. Biol. Chem. 250, 27832788 (1975)

3. Evans, M. C. W.: Electron paramagnetic resonance studies in photosynthesis. In: Primary Processes of Photosynthesis (J. Barber, ed.). Elsevier/North-Holland, Biomedical Press, Amsterdam, pp. 433-464 (1977)

4. Evans, M. C. W. \& R. Cammack: The effect of the redox state of the bound iron-sulphur centres in spinach chloroplasts on the reversibility of P700 photooxidation at low temperatures. Biochem. Biophys. Res. Commun. 63, 187-193 (1975)

5. Evans, M. C. W. \& P. Heathcote: Effects of glycerol on the redox properties of the electron acceptor complex in spinach photosystem I particles. Biochim. Biophys. Acta 590, 89-96 (1980)

6. Evans, M. C. W., C. K. Sihra \& R. Cammack: The properties of the primary electron acceptor in the photosystem I reaction centre of spinach chloroplasts and its interaction with P700 and the bound ferredoxin in various oxidationreduction states. Biochem. 3. 158, 71-77 (1976)

7. Heathcote, P.\& M. C. W. Evans: Properties of the EPR spectrum of the intermediary electron acceptor $\left(A_{1}\right)$ in several different photosystem I particle preparations. FEBS Lett. 111, 381-385 (1980)

8. Heathcote, P., K. N. Timofeev \& M. C. W. Evans: Detection by EPR spectrometry of a new intermediate in the primary photochemistry of photosystem I particles isolated using Triton X-100. FEBS Lett. 101, 105-109 (1979)

9. Hiller, R. G., B. L. Møller \& G. HøyerHANSEN: Characterization of six putative photosystem I mutants in barley. Carlsberg Res. Commun. 45, 315-328 (1980)

10. Hoyer-Hansen, G. \& D. J. Simpson: Changes in the polypeptide composition of internal membranes of barley plastids during greening. Carlsberg Res. Commun. 42, 379-389 (1977)

11. Johanningmeier, U., P. V. Sane, G. HøyerHaNSEN \& B. L. Møller: The inhibition of 
photosynthetic electron flow by $N, N^{\prime}$-dicyclohexylcarbodiimide (DCCD). In: Proc. Int. Congr. Photosynth. Sth (G. Akoyunoglou ed.) in press (1981)

12. Malkin, R. \& H. B. Posner: On the site of function of the Rieske iron-sulfur center in the chloroplast electron transport chain. Biochim. Biophys. Acta 501, 552-554 (1978)

13. Malkin, R. \& T. Vanngard: An EPR study of cytochromes from spinach chloroplasts. FEBS Lett. 111, 228-231 (1980)

14. Mathis, P., K. Sauer \& R. Remy: Rapidly reversible flash-induced electron transfer in a $P 700$ chlorophyll-protein complex isolated with SDS. FEBS Lett. 88, 275-278 (1978)

15. Metz, J. G., J. Wong \& N. Bishop: Changes in electrophoretic mobility of a chloroplast membrane polypeptide associated with the loss of the oxidizing side of photosystem II in low fluorescence mutants of Scenedesmus. FEBS Lett. $114,61-66$ (1980)

16. Møller, B. L., G. Høyer-Hansen \& R. G. HiLLER: Functional identification of barley thylakoid polypeptides resolved by SDS-polyacrylamide gel electrophoresis. In: Proc. Int. Congr. Photosynth. 5th. (G. Akoyunoglou, ed.) in press (1981)

17. Møller, B. L., R. M. Smillie \& G. HøyerHANSEN: A photosystem I mutant in barley (Hordeum vulgare L.). Carlsberg Res. Commun. 45 , $87-99(1980)$

18. Nelson, N., C. Bengis, B. L. Silver, D. Getz \& M. C. W. Evans: Electron spin resonance studies of bound ferredoxin in chloroplast photosystem I reaction center. FEBS Lett. 58, 363-365 (1975)

19. Nugent, J. H. A. \& M. C. W. Evans: Lightinduced EPR signals at cryogenic temperatures in subchloroplast particles enriched in photosystem II. FEBS Lett. 101, 101-104 (1979)
20. Nugent, J. H. A. \& M. C. W. Evans: EPR signals of cytochromes in subchloroplast particles, FEBS Lett. 112, 1-4 (1980)

21. Nugent, J. H. A., M. C. W. Evans \& B. L. MøLlER: Detection of photosystem II reaction centre components by electron paramagnetic resonance spectroscopy. In: Proc. Int. Congr. Photosynth. 5th (G. Akoyunoglou, ed.) in press 1981

22. Nugent, J. H. A., B. L. Møller \& M. C. W. EvaNS: EPR detection of the primary photochemistry of photosystem II in a barley mutant lacking photosystem I activity. FEBS Lett. 121, 355-357 (1980)

23. Nugent, J. H. A., B. L. Møller \& M. C. W. Evans: Comparison of the EPR properties of photosystem I iron-sulphur centres $A$ and $B$ in spinach and barley. Biochim. Biophys. Acta, 634, 249-255 (1981)

24. OHNISHI, T.: Mitochondrial iron sulphur flavodehydrogenases. In: Membrane proteins in energy transduction (R. A. Capaldi, ed.) Marcel Dekker Inc. pp 1-87 (1979)

25. Prince, R. C., M. S. Crowder \& A. J. Bearden: The orientation of the magnetic axes of the membrane-bound iron-sulphur clusters of spinach chloroplasts. Biochim. Biophys. Acta, 592, 323-337 (1980)

26. Shuvalov, V. A., E. Dolan \& B. KE: Spectral and kinetic evidence for two early electron acceptors in photosystem I. Proc. Natl. Acad. Sci. USA, 76, 770-773 (1979)

27. Wettstein, D. von, B. L. Møller, G. HøyerHansen \& D. Simpson: Mutants in the analysis of the photosynthetic membrane polypeptides. In: Origin of chloroplasts (J. A. Schiff and R. Y. Stanier, eds.). Elsevier/North-Holland, Biomedical Press, Amsterdam. In press (1980) 